\title{
DERECHO Y POLÍTICAS AMBIENTALES EN ARAGÓN (SEGUNDO SEMESTRE 2019)(*)
}

\author{
OLGA HERRÁIZ SERRANO \\ Letrada de las Cortes de Aragón \\ Profesora Asociada Doctora de Derecho Administrativo de la \\ Universidad de Zaragoza
} ADESTER (Estudios Territoriales), reconocido por el Gobierno de Aragón (BOA núm. 48, de 20 de abril de 2005).

[DOI: http://dx.doi.org/10.17345/rcda.2693] 
Sumario: 1. Introducción. 2. La nueva administración ambiental aragonesa tras las elecciones celebradas en mayo de 2019. 3. Legislación. 4. Otras disposiciones. 5. Medidas de fomento de carácter ambiental.

\section{INTRODUCCIÓN}

Dado que esta crónica se dirige a dar cuenta de la política ambiental de la Comunidad Autónoma de Aragón en el semestre comprendido entre abril y septiembre, incluido, de 2019, se entenderá fácilmente que el protagonismo lo tengan los resultados de las elecciones autonómicas celebradas en mayo y la conformación del nuevo Gobierno y, con él, la estructura de la Administración ambiental aragonesa para la $X$ Legislatura, a que se ha dado inicio. En el apartado legislativo, daremos cuenta de la aprobación de alguna disposición reglamentaria de menor nivel a la espera de que el nuevo Departamento con competencias en materia de medioambiente recupere la normalidad tras las renovaciones oportunas de sus órganos directivos.

Entrando en la reseña de las elecciones autonómicas, con una participación ligeramente inferior a la de los comicios precedentes de 2015 (66,16\% frente al 68,27\%), el PSOE ganó las elecciones en Aragón al obtener el 30,8\% de los votos (206.400) frente al 20,8\% del PP (139.660), al 16,6\% de CiudadanosPartido de la Ciudadanía (111.602), al 8,1\% de Podemos-Equo (54.252), al $6,2 \%$ de Chunta Aragonesista (41.879), al 6,08\% de VOX (40.671), al 5,08\% del Partido Aragonés (33.978), y al 3,32 de Izquierda Unida de Aragón (22.229). En escaños, el PSOE obtuvo 24 frente a los 18 de 2015. El PP bajó de 21 a 16 y Podemos Aragón cayó a 5 desde sus 14 anteriores. CiudadanosPartido de la Ciudadanía (C'S) subió de 5 escaños a 12, mientras que Chunta Aragonesista, el Partido Aragonés (PAR) y VOX, que irrumpe por primera vez en la Cámara, obtuvieron los mismos 3 escaños, repitiendo, con tan solo 1 , Izquierda Unida de Aragón. Así, si las elecciones autonómicas de 2015 habían supuesto el paso de cinco a siete partidos políticos o coaliciones electorales en las Cortes de Aragón, en la actual Cámara aragonesa nos encontramos con ocho formaciones políticas y otros tantos grupos parlamentarios. En definitiva, no solo se confirma la complejidad del sistema de partidos de la pasada legislatura, sino que se incrementa, con la consiguiente dificultad para alcanzar acuerdos. 
La primera manifestación de ello la tuvimos con la gestación del que propició la reelección del líder del Partido Socialista, Javier Lambán, como presidente del Gobierno de Aragón en la sesión de investidura celebrada los días 30 y 31 de julio de 2019, con los votos a favor de su Grupo, además de Podemos-Equo, Chunta Aragonesista, Partido Aragonés e Izquierda Unida de Aragón (36 votos) frente a 31 en contra. Así las cosas, al día siguiente de las elecciones, con los fríos números en la mano, parecían más probables otras dos coaliciones de gobierno: la de las tres fuerzas del centro-derecha cuyos votos sumaban 340 la del Partido Socialista con Ciudadanos-Partido de la Ciudadanía que, juntos, sumaban 36 votos. Sin embargo, un elemento inesperado marcó el rumbo de los acontecimientos. El Partido Socialista dijo haber suscrito un acuerdo con el Partido Aragonés, que hacía inviable la primera de las opciones, pero que a la postre también habría de dificultar la segunda, en un caso por las diferencias ideológicas irreconciliables entre el centralismo de VOX y la férrea defensa del Estado autonómico del PAR y, en otro, por los distintos planteamientos de estructura territorial entre este último y Ciudadanos. Por su parte, la inclusión en la coalición de gobierno que finalmente propició la investidura de fuerzas ideológicamente tan dispares y tan enfrentadas en el pasado reciente de nuestra Comunidad Autónoma, como Podemos-Equo y el Partido Aragonés, resulto en sí misma sorprendente. Pero es un hecho que estos dos partidos, el Partido Socialista y Chunta Aragonesista formalizaron el 1 de agosto de 2019 un documento que, bajo el título de Acuerdo de investidura y gobernabilidad para la X Legislatura en Aragón, recogía los 132 compromisos alcanzados entre ellos, que se sistematizaban en bloques temáticos.

A los efectos del presente trabajo, destacaremos el cuarto bloque temático del Acuerdo de investidura y gobernabilidad, rubricado "Energía, medio ambiente y agricultura", que contenía 30 prioridades, la mayoría de las cuales caen de lleno en la órbita del medio ambiente y contemplan mandatos asumidos por este ejecutivo de coalición y de cuyo cumplimiento habrá de dar cuenta en los próximos cuatro años. Nos referimos a los siguientes:

\section{"ENERGÍA}

87. Impulsar una transición energética justa, implementando la Estrategia Aragonesa contra el Cambio Climático e impulsar una Ley aragonesa de 
cambio climático y transición energética que contenga las medidas y planes necesarios para que, los aragoneses que se vean directamente afectados por la misma, no vean mermadas sus condiciones de vida y el empleo.

88. Mantener el liderazgo de Aragón en energías renovables.

89. Impulsar la reversión de las concesiones hidroeléctricas que caducan para la gestión pública con beneficio directo a las subcuencas afectadas, así como la entrega de la energía reservada.

90. Desarrollar soluciones para las Cuencas Mineras en consonancia con el Dictamen de la Comisión Especial de Estudio para analizar los criterios que deben regir la transición energética en Aragón y, en particular, en lo concerniente a la continuidad de la central térmica de Andorra, que incluya una estrategia de desarrollo socioeconómico alternativo y un estudio de viabilidad del mismo aprobado por las Cortes de Aragón.

91. Fomentar la rehabilitación energética para la eficiencia en edificios de la administración pública aragonesa y apoyar la financiación de proyectos de renovables en el sector residencial, especialmente hacia la población con menos recursos, para favorecer su abastecimiento.

92. Poner en marcha una red pública de puntos de recarga para vehículos eléctricos en colaboración con otras administraciones.

\section{MATERIA HIDROLÓGICA}

93. Defender del principio de unidad de cuenca, y cumplimiento de la Directiva Marco del Agua, la defensa de la reserva hídrica y manifestar nuestra oposición rotunda a cualquier Trasvase del río Ebro.

94. Trabajar por la resolución de los conflictos del Agua en Aragón. Constitución e impulso de una mesa de diálogo en la Comunidad Autónoma de Aragón para un nuevo Acuerdo del Agua. Asegurando el desarrollo de las necesarias infraestructuras de nuestro territorio para el progreso de nuestro medio rural con el agua como recurso vertebrador, de generación de riqueza y oportunidades y de cohesión del territorio. Todo ello $\sin$ olvidar las restituciones justas a afectados por embalses 
ejecutados o no. De igual modo, se desarrollarán las conclusiones y recomendaciones para los usos del agua aprobados en la subcomisión de Agua y Cambio Climático del Congreso de los Diputados.

95. Acometer la limpieza de aquellos cauces de ríos que resulten urgentes ya sea por motivos de inundaciones o avenidas de acuerdo con el Dictamen de la Ponencia de Inundaciones de la Comisión del Agua de Aragón: y el Plan Integral de Gestión del Riesgo de Inundación de la Confederación Hidrográfica del Ebro.

96. Adoptar las medidas necesarias para poner en marcha el Plan Ebro 2030. Impulsar la zona de la Ribera con compra de terrenos para zonas inundables, limpieza ecológica del río, plantación de 3 millones de árboles y una red de infraestructuras para convertir a la zona como un gran corredor de economías verdes basadas en el conocimiento y en el ecosistema.

97. Implementar los trabajos y normativas que aseguren la calidad de las masas de agua, y la descontaminación de aquellas que se encuentren en mal estado. Realizar acciones conjuntas con la $\mathrm{CHE}$.

98. Fomentar la modernización de regadíos con apuesta por regadíos sociales sostenibles de acuerdo con el Inventario de Regadíos.

\section{MEDIO AMBIENTE Y EMERGENCIA CLIMÁTICA}

99. Desarrollar la Ley 15/2006, de 28 de diciembre, de Montes de Aragón especialmente en lo que respecta al aumento del período de contratación de los profesionales del operativo y la mejora sustancial de medios y herramientas de trabajo.

100. Preservar la biodiversidad terrestre y acuática y aumentar la dotación de los Espacios naturales protegidos, sobre todo las del Parque Nacional de Ordesa y Monte Perdido.

101. Impulsar el desarrollo de las Comarcas de Montaña a través de la formulación de las Directrices Territoriales recogidas en el Texto Refundido de la Ley de Ordenación del Territorio de Aragón. 
102. Aprobar y ejecutar el Plan Forestal de Aragón, con estrategias autonómicas y actuaciones priorizadas de tratamientos de masas, lucha contra la desertificación, plantaciones y puesta en valor de la masa vegetal.

103. Desarrollar un Plan de Acción contra la Desertificación de Aragón y elaboración de un Inventario aragonés de Erosión de Suelos.

104. Coordinar y mejorar los recursos para la descontaminación de suelos.

105. Fomentar el Residuo Cero mediante la recuperación de materia orgánica, retorno de envases y legislación contra plásticos y objetos de un solo uso, entre otras medidas".

\section{LA NUEVA ADMINISTRACIÓN AMBIENTAL ARAGONESA TRAS LAS ELECCIONES CELEBRADAS EN MAYO DE 2019}

Celebradas las elecciones autonómicas en mayo de 2019 e investido nuevo Presidente de la Comunidad Autónoma el líder del Partido Socialista, con el apoyo de Podemos Aragón, el Partido Aragonés y Chunta Aragonesista, la duda desde el punto de vista de la organización de la Administración ambiental aragonesa era si se mantendrían las competencias agrupadas con las correspondientes al sector agropecuario en un único departamento, como había sucedido en la VIII y IX legislaturas, o si se volvería a crear una consejería propia en materia medioambiental, como también había ocurrido entre 1993 y 1995, y de forma ininterrumpida entre 1999 y 2011. Así las cosas, el Decreto de 5 de agosto de 2019, del Presidente del Gobierno de Aragón, por el que se modifica la organización de la Administración de la Comunidad Autónoma de Aragón y se asignan competencias a los Departamentos, y el Decreto 93/2019, de 8 de agosto, del Gobierno de Aragón, por el que se desarrolla la estructura orgánica básica de la Administración de la Comunidad Autónoma de Aragón (publicados en BOA de 6 y 9 de agosto, respectivamente) despejaron la incógnita. Se optó por la continuidad inmediata de la estructura anterior, aumentando de cinco a seis el número de las direcciones generales del, eso sí, rebautizado como Departamento de Agricultura, Ganadería y Medio Ambiente, al que se le atribuyeron la totalidad de las competencias que venían asignadas al Departamento de Desarrollo Rural y Sostenibilidad. 
Como detalla el Decreto 93/2019, la estructura orgánica de la nueva consejería quedaba del siguiente modo: Secretaría General Técnica; Dirección General de Producción Agraria; Dirección General de Desarrollo Rural; Dirección General de Calidad y Seguridad Alimentaria; Dirección General de Innovación y Promoción Agroalimentaria (desdoblándose así la anterior Dirección General de Alimentación y Fomento Agroalimentario); Dirección General de Medio Natural y Gestión Forestal, y Dirección General de Cambio Climático y Educación Ambiental (estas dos últimas en sustitución de la Dirección General de Gestión Forestal, Caza y Pesca y de la Dirección General de Sostenibilidad). Precisamente, por medio de la Orden AGM/1088/2019, de 29 de agosto (BOA 5 septiembre), se determinó que, transitoriamente (hasta tanto se aprobase el decreto de estructura orgánica de la consejería), las competencias que tenían atribuidas la Dirección General de Gestión Forestal, Caza y Pesca (a excepción de las correspondientes a estas dos últimas materias) y la Dirección General de Sostenibilidad en materia de biodiversidad, espacios naturales y Red Natura 2000 fuesen asumidas por la Dirección General de Medio Natural y Gestión Forestal, que continuará ejerciéndolas a través de los servicios y unidades administrativas adscritos a las dos direcciones generales suprimidas. Por su parte, la Dirección General de Cambio Climático y Educación Ambiental asume las competencias de la Dirección General de Sostenibilidad en materia de cambio climático, educación y planificación ambiental, así como la Unidad para la Descontaminación Integral del Lindano. Además, la Dirección General de Calidad y Seguridad Alimentaria incluye las competencias de la extinta Dirección General de Alimentación y Fomento Agroalimentario correspondientes a los Servicios de Sanidad Animal y Vegetal y de Seguridad Agroalimentaria. También asume las competencias de la desaparecida Dirección General de Gestión Forestal, Caza y Pesca relativas a estas últimas, así como las de la Dirección General de Sostenibilidad referentes al control ambiental. Finalmente, a la Dirección General de Innovación y Promoción Agroalimentaria le van a corresponder las competencias de la Dirección General de Alimentación y Fomento Agroalimentario en materia de industrialización agroalimentaria y de promoción y calidad agroalimentaria. 
Todo ello tendrá que adquirir carta de naturaleza en la disposición que modifique el Decreto 317/2015, de 15 de diciembre, del Gobierno de Aragón, por el que se aprobó la estructura orgánica del Departamento de Desarrollo Rural y Sostenibilidad, pero transitoriamente las competencias atribuidas a los órganos directivos del Departamento de Agricultura, Ganadería y Medio Ambiente, creados por el Decreto 93/2019, de 8 de agosto, del Gobierno de Aragón, se ejercitarán a través de los Servicios y Unidades que por lo que respecta a las tres direcciones con atribuciones ambientales son los siguientes: Servicio de Sanidad Animal y Vegetal, Servicio de Seguridad Agroalimentaria, Servicio de Control Ambiental y Servicio de Caza, Pesca y Medio Acuático, Centro de Sanidad y Certificación Vegetal y Laboratorio Agroambiental en la Dirección General de Calidad y Seguridad Alimentaria; Servicio de Planificación y Gestión Forestal, Servicio de Gestión de los Incendios Forestales y Coordinación, Servicio de Biodiversidad y Servicio de Espacios Naturales y Red Natura 2000 en la Dirección General de Medio Natural y Gestión Foresta; y, finalmente, Servicio de Planificación Ambiental, Servicio de Cambio Climático y Educación Ambiental y Unidad para la Descontaminación Integral del Lindano en la Dirección General de Cambio Climático y Educación Ambiental.

Por otro lado, siguen adscritas al departamento con competencias en materia agrícola, ganadera y medio ambiental las entidades de derecho público Instituto Aragonés del Agua (IAA) e Instituto Aragonés de Gestión Ambiental (INAGA), así como las sociedades Sociedad Aragonesa de Gestión Agroambiental, S.L.U. y Aragonesa de Gestión de Residuos, S.A. Subrayaremos que el cuatripartito apuesta por la continuidad del IAA, un instituto para el que, en la pasada legislatura, el Gobierno de Lambán llegó a remitir un proyecto de ley a las Cortes que, con el eufemístico título de reorganización de competencias administrativas en materia de aguas, pretendía llevar a efecto la extinción de aquella entidad y que sus funciones fuesen asumidas de nuevo por una dirección general del entonces Departamento de Desarrollo Rural y Sostenibilidad. La tramitación de dicha iniciativa legislativa se encalló por las diferencias precisamente con Podemos Aragón y, seguramente por eso, la misma no figura entre las 132 prioridades del acuerdo de gobernabilidad suscrito por los cuatro partidos que sostienen al actual ejecutivo aragonés. 
Finalmente, en lo que concierne a la Administración periférica del departamento, se mantiene la ya consolidada, que consta en el Decreto 142/2012, de 22 de mayo, por lo que se compone de los Servicios Provinciales de Agricultura, Ganadería y Medio Ambiente en Huesca, Teruel y Zaragoza, directamente dependientes del Consejero, que ejercerán sus competencias sin perjuicio de las funciones de dirección, impulso, coordinación y supervisión de los servicios que tienen atribuidas, en sus respectivas provincias, las Delegaciones Territoriales del Gobierno de Aragón en Huesca y Teruel. Para prestar su actividad en el ámbito inferior al provincial el Departamento de Agricultura, Ganadería y Medio Ambiente dispondrá de un conjunto de Oficinas Comarcales de Agricultura y Alimentación (OCAS) y de Áreas Medioambientales, cuyo ámbito territorial coincide con el de las comarcas aragonesas.

La continuidad básica que, en la recién estrenada $X$ Legislatura, presenta la estructura de la Administración ambiental aragonesa tiene su reflejo también en la persona titular del departamento, pues, tras el cese de don Joaquín Olona Blasco como consejero en funciones de Desarrollo Rural y Sostenibilidad, fue nombrado él mismo Consejero de Agricultura, Ganadería y Medio Ambiente (sendos Decretos de 5 de agosto de 2019, del Presidente del Gobierno de Aragón, BOA 6 agosto). No obstante, la complejidad de esta legislatura, con un cuatripartito al frente de la DGA, se refleja en la convivencia en una misma consejería de altos cargos de distinto color político. En el caso de la que nos ocupa, responsables de Podemos-Equo gestionarán dos direcciones generales: Medio Natural y Gestión Forestal, y Cambio Climático y Educación Ambiental.

Por terminar con el capítulo organizativo, citaremos la creación del Consejo Aragonés del Clima, como órgano colegiado de carácter consultivo con el propósito de "reforzar la gobernanza del cambio climático, impulsar las medidas de mitigación y adaptación al cambio climático en la Comunidad Autónoma de Aragón, así como el seguimiento de la Estrategia Aragonesa de Cambio Climático-Horizonte 2030" (Decreto 79/2019, de 21 de mayo, BOA 29 mayo). Conformado por una Presidencia y una Vicepresidencia, que recaerán, respectivamente, en el titular del departamento y de la dirección general con 
competencias en materia de cambio climático, contará también con un máximo de 27 vocalías y una secretaría. Se trata de un órgano colegiado de los que se dicen representativos de intereses sociales pues, entre los vocales, figuran no solo representantes de diversas Administraciones sino, además, de organizaciones sindicales, empresariales, conservacionistas y profesionales. El mandato de las personas que sean designadas como titulares de las vocalías del Consejo Aragonés del Clima tendrá una duración de dos años, siendo renovados automáticamente por idéntico periodo de tiempo si no fuesen revocados.

\section{LEGISLACIÓN}

Como se ha anticipado, al estar comprendido el período que analizamos entre la disolución de las Cortes de Aragón con motivo de la convocatoria de elecciones y la reciente puesta en marcha del gobierno surgido de las urnas, el apartado legislativo se ve considerablemente mermado. Tanto es así que solo destacaremos la aprobación de una disposición de carácter reglamentario como es el Decreto 53/2019, de 26 de marzo, por el que se regula la gestión de estiércoles y los procedimientos de acreditación y control.

Para contextualizarlo, es preciso recordar que la disposición adicional quinta de la Ley 11/2014, de 4 de diciembre, de Prevención y Protección Ambiental de Aragón, obliga a las explotaciones ganaderas intensivas sujetas al régimen de autorización ambiental integrada a acreditar, para la obtención o revisión de la misma, que los procesos de gestión de los estiércoles que producen cumplen los requisitos establecidos en la normativa sectorial (básicamente, normativa sobre contaminación de agua, residuos, emisiones a la atmósfera, directrices ganaderas y fertilización), obligación que también extiende a aquellas explotaciones ganaderas que, por sus dimensiones y características, no estuviesen sujetas a autorización ambiental integrada, sino a mera licencia ambiental de actividades clasificadas. La misma disposición definió qué ha de entenderse legalmente por aplicación directa de estiércoles en la agricultura y estableció los sistemas que pueden utilizar los promotores de las explotaciones ganaderas para gestionarlos correctamente. Permitiéndoles optar por aplicarlos directamente en la agricultura sin tratamiento o someterlos a uno, a su vez el tratamiento podrá ser realizado en centros de gestión de estiércoles o en la 
propia instalación por el productor, o mediante una combinación de dos o más de los sistemas anteriores. En cualquier caso, la correcta gestión de aquellos exigía un desarrollo reglamentario para el que la ley concedía un año, pero que el Gobierno aragonés ha excedido con creces, incluso después de conocerse las conclusiones de la Comisión Europea sobre las mejores técnicas disponibles (MTD) respecto a la cría intensiva de aves de corral y de cerdos.

En efecto, la Decisión de Ejecución (UE) 2017/302, de 15 de febrero, concede un plazo de cuatro años a partir de su publicación para que se adapten todas las autorizaciones ambientales integradas otorgadas a dichas actividades. Para concretar las exigencias de dicha adaptación y dar cumplimiento al mandato contenido en la disposición adicional quinta de la Ley 11/2014, el Gobierno de Aragón aprobó el Decreto 53/2019, de 26 de marzo, por el que se regula la gestión de estiércoles y los procedimientos de acreditación y control (BOA 8 abril). Por un lado, dicha disposición regula el modelo de autogestión, definido como el supuesto en el que el ganadero productor asume la gestión de sus estiércoles y, por otro, regula el modelo de centros de gestión, entendidos como entidades no titulares de explotaciones ganaderas que prestan el servicio consistente en la aceptación del estiércol y de las responsabilidades de su gestión.

Para el caso de que el ganadero destine el estiércol a su utilización como fertilizante agrícola bajo el modelo de autogestión, el nuevo procedimiento de control completa el régimen clásico de adscripción de las parcelas agrícolas a las granjas mediante un sistema basado en declaraciones anuales del ganadero, en las que se identifican las cantidades de estiércol aplicadas a cada parcela en cada campaña agrícola. Las declaraciones quedan así adaptadas a las necesidades de la explotación agrícola, conforme a la demanda de los distintos cultivos en cada campaña agrícola.

Para el caso de que el ganadero productor opte por la entrega de todos los estiércoles a los centros de gestión, el sistema de declaraciones anuales se limita a precisar las cantidades de estiércol entregadas a los centros de gestión y a identificar los centros que los han aceptado. El sistema de declaraciones anuales también se adapta al supuesto de que el ganadero opte por una 
combinación entre la autogestión de una parte del estiércol y la entrega del resto a los centros de gestión.

También en cumplimiento de la disposición adicional quinta de la Ley de Prevención y Protección Ambiental de Aragón, el Decreto 53/2019 desarrolla el procedimiento y condiciones de autorización de los centros de gestión de estiércoles. Puesto que estos aceptan las responsabilidades medioambientales propias de la posesión o gestión de los estiércoles, de conformidad con la Ley $11 / 2014$, quedan sujetos al régimen de intervención ambiental de licencia de actividades clasificadas, sin perjuicio de su sometimiento al régimen de autorización ambiental integrada cuando realicen actividades contempladas en el anexo IV de aquella, debiendo cumplir asimismo el régimen de autorización y registro establecido en el Reglamento (CE) $n . \stackrel{0}{1069 / 2009}$, del Parlamento Europeo y del Consejo, de 21 de octubre, por el que se establecen las normas sanitarias aplicables a los subproductos animales y los productos derivados no destinados al consumo humano y por el que se deroga el Reglamento (CE) n.ำ $1774 / 2002$.

El régimen de inspección y control público al que se someten las explotaciones ganaderas y los centros de gestión de estiércoles es sustancialmente distinto en función del instrumento de control ambiental que les resulte de aplicación. También es distinta la competencia para el ejercicio de las funciones de inspección, y de sanción en caso de incumplimiento, ya que el control de las explotaciones y centros sujetos al régimen de licencia ambiental de actividades clasificadas es competencia de los ayuntamientos, mientras que la competencia de control de las explotaciones de los centros de gestión sujetos al régimen de autorización ambiental integrada corresponde al Gobierno de Aragón. Precisamente por ello y para tratar de asegurar la coherencia y la homogeneidad en la acción pública de control, el Decreto 53/2009 exige que se apruebe, mediante Orden del consejero o consejeros competentes en materia de agricultura, de ganadería y de medio ambiente, el Plan de Inspección y Control, que deberá afectar a todas las actividades de producción y gestión de estiércoles y residuos orgánicos que compiten en la oferta de fertilizantes nitrogenados orgánicos, incluyendo en su ámbito de aplicación a todas las explotaciones ganaderas y centros gestores de estiércoles, sin excepción de los sujetos al régimen de licencia ambiental de actividades clasificadas. El Plan 
de Inspección y Control también será vinculante para los entes locales que otorgaron las licencias ambientales de actividades clasificadas a explotaciones ganaderas y centros de gestión que se encuentren vigentes, sin perjuicio de que cada entidad local pueda aprobar un plan que complemente al autonómico.

\section{OTRAS DISPOSICIONES}

- Orden DRS/330/2019, de 26 de marzo, por la que se actualizan varios anexos de las Directrices sectoriales sobre actividades e instalaciones ganaderas, cuya revisión se aprobó por el Decreto 94/2009, de 26 de mayo, del Gobierno de Aragón (BOA 8 abril).

- Orden DRS/333/2019, de 25 de marzo, por la que se declaran, en base al índice de carga ganadera, los municipios con sobrecarga ganadera por exceso de nitrógeno de origen orgánico procedente de la actividad ganadera (BOA 8 abril).

- ORDEN DRS/882/2019, de 8 de julio, por la que se designan y modifican las zonas vulnerables a la contaminación de las aguas por nitratos procedentes de fuentes agrarias en la Comunidad Autónoma de Aragón (BOA 25 julio y corrección errores 26 agosto).

- Orden VMV/358/2019, de 28 de marzo, por la que se establecen los criterios que permiten la caracterización como recursos turísticos de determinados itinerarios senderistas (BOA 16 abril), entre los que son tenidos en cuenta los de localización en el medio natural, por la calidad del mismo y del paisaje.

\section{MEDIDAS DE FOMENTO DE CARÁCTER AMBIENTAL}

- Decreto 60/2019, de 9 de abril, del Gobierno de Aragón, por el que se otorga el Premio Aragón 2019 (BOA 15 abril).

- Orden DRS/831/2019, de 27 de junio, por la que se convocan subvenciones para la adaptación de la ganadería extensiva a los retos ambientales y a los desafíos socioterritoriales para el año 2019 (BOA 17 julio).

- Orden DRS/956/2019, de 25 de julio, por la que se convocan subvenciones públicas en el área de influencia socio-económica del Parque 
Nacional de Ordesa y Monte Perdido, de acuerdo con lo previsto en el Real Decreto 1229/2005, de 13 de octubre, para el año 2019 (BOA 9 agosto). 